\title{
Melt Volume Flow Measurement in the Mineral-Wool Production Process
}

\author{
Jinpeng Chen ${ }^{1}$ - Marko Hočevar2,* - Brane Šrrok² \\ ${ }^{1}$ Lanzhou Polytechnical College, Faculty of Electrical Engineering, Kitajska \\ 2 University of Ljubljana, Faculty of Mechanical Engineering, Slovenija
}

\begin{abstract}
The quality of the end product in mineral wool production depends on the fiberisation process on the spinning machine. An important process variable is the fluctuation of melt volume flow from the melter. In this paper, a method for measuring melt volume flow, which is based on the measuring the diameter and flow velocity using machine vision, is presented. The velocity is measured by measuring the time of the travel of flow disturbances on two consecutive images using a correlation method. The flow disturbances are generated using a pneumatic system. The results of laboratory calibration using water as well as the results of field measurements in a mineral wool plant are presented. The field measurements were performed by comparison with the weight of the final product. The results of laboratory calibration show agreement with reference volume flow measurements with $R^{2}=0.96$ for water. The agreement among field measured values and available comparison method is also very good.

(C2011 Journal of Mechanical Engineering. All rights reserved.
\end{abstract}

Keywords: mineral wool, melt, volume flow measurement, machine vision

\section{INTRODUCTION}

Mineral-wool is a general name given to many inorganic insulation materials made of fibres. It is widely used in the industry owing to its excellent properties in sound- and thermal insulation. There are several production methods for mineral wool [1]. The most commonly used mineral-wool production process is the fiberisation process of molten rock, which melts in a cupola or tub furnace on quickly rotating spinning discs. Molten rock enters through a siphon neck into a homogenization reservoir. Over the weir and directing channel, the molten rock falls under gravity onto the rotating discs of the spinning machine. Fabrication of mineral wool occurs on the spinning machine, as the melt droplets separate from the rotating wheels and are fiberised by high-speed airflow around the wheels. Using high-speed airflow that is fed coaxially over the disc, fibres are transported away from the spinning machine and thrown into a forming chamber. After leaving it, mineral wool enters the secondary conveyor belt where it is thermally treated in the polymerization chamber and finalised/cut to selected density, thickness and size.
The continuous melt volume flow measurement is desired for the regulation of the melter operation and for the regulation of the velocity of mineral wool product line. Melt volume flow fluctuations directly affect the quality of the final product of mineral-wool. Continuous melt volume flow measurements enable the regulation of product line velocity, which is important for compensating the density variations in the final product due to variations of melt volume flow from the melter.

Measurements of melt volume flow using conventional methods are not possible due to very high temperature of the melt. Temperature of the melt usually ranges from 1380 to 1500 ${ }^{\circ} \mathrm{C}$, and melt dynamic viscosity $\mu$ from 15 to $8 \mathrm{dPa} \cdot \mathrm{s}$, surface tension $\sigma$ from 0.439 to 0.410 $\mathrm{N} / \mathrm{m}$, and range of volume flows from 3 to 5.5 $\mathrm{t} / \mathrm{h}$. Currently, no reliable measurement method capable of instantaneous measurements of melt volume flow from the melter, is available. The following methods are used with limited success: measurement of the current consumed by the spinning machine electric motors [2], volume flow measurement using radioactive isotopes, weighing of primary wool layer [3] and [4], and weighing of the final product. The current consumed by the spinning machine electric motors depends on

*Corr. Author's Address: University of Ljubljana, Faculty of Mechanical Engineering,

Aškerčeva 6, 1000 Ljubljana, Slovenia, marko.hocevar@fs.uni-lj.si 
the melt volume flow as well as on the melt flow impingement point position, vibrations of wheels, etc. Volume flow measurement by radioactive isotopes is not used anymore due to safety and environmental concerns. Weighing of primary wool layer has proved unreliable due to a very low ratio of weight of the primary wool layer and weighing device, deposition of dirt and vibrations. The measurement results of weighing the final product are only available several minutes later, which is not suitable for production line speed control.

In this paper, a new method for measuring the volume flow of the melt in front of the spinning machine (Fig. 1) in the process of mineral wool production is presented. The volume flow is calculated from the measured values of diameter and velocity of melt flow. Due to very high temperatures, these variables are measured by acquiring images with industrial camera and by image analysis. Diameter is measured by detecting the border of melt flow, while velocity is measured from the measurement of time of travel of flow disturbances on two consecutive images using correlation method.

The method of velocity measurements is similar to flow measurement methods based on correlation and particle image velocimetry (PIV) [5]. A review of the PIV method development is given in [5] and [6]. PIV method enables quantitative measurement of fluid velocity vectors at a very large number of points simultaneously. Research focuses on the correlation of two successive scalar gray intensity images for the purpose of measuring imaged fluid motions. Authors employ various techniques of image processing among which are locally correlates images for displacements, rotations, deformations, and higher-order displacement gradient fields [7]. For a high amount of details on images, particle image analysis method is preferred in relation to particle tracking method [8]. Application of PIV is broad, Cheng et al. [8] algorithm was based on computing the cross correlation of bubble images with multiple spatial resolutions for bubbles flow in the tank, Zosel et al. [9] used $\mu$ PIV for measurement of flow in micro holes and Hann et al. [10] used PIV for the measurement of flow velocity and acoustic particle velocity in a standing wave tube. Among measurements of jets, Patte-Rouland et al. [11] used PIV method with orthogonal decomposition of a jet in the recirculation region, Hui Hu et al. [12] studied the mixing process in a lobed jet flow, and Sakakibara et al. [13] performed measurements of the particle/ fluid velocity correlation and the dissipation of turbulent kinetic energy by particles. Cross correlation techniques are also used for flow velocity and volume flow rates measurement with other measurement principles [14]. Among those, Carlson and Ing [15] used ultrasonic speckle correlation imaging for flow velocity measurement in a vertical pipe, and Takamoto et al. [16] used ultrasonic correlation technique for measurements for very low volume flow rates.

The disturbances are detected if they are already present in the flow or if they are generated using a pneumatic disturbance generation system. Disturbance is generated using an airflow nozzle, mounted on a movable pneumatic cylinder, which enables positioning near the melt flow at the time of disturbance generation. The airflow nozzle is opened by a electric valve for several short bursts at the selected time intervals. Disturbance generation should not be repeated too often, e.g. interval too short as this increases the possibility of spilling the melt and disturbing the production process. Diameter can be continuously measured during the production process, while the measurement of velocity using the disturbance method can only be performed when disturbance is triggered. For proper process control, however, continuous volume flow estimation including velocity measurement of flow is required. Therefore, a contraction method for estimating small changes in velocity in real time when the velocity measurement using disturbance method is not available has been introduced. The principle of the contraction method is the following: the higher the flow velocity, the less contraction in the flow jet there is, and the lower the flow velocity, the more contraction in the flow jet there is.

The calibration of the measurement method in the production process is difficult since there is no reliable comparison method. Most appropriate is the comparison with weighing the material in front of the polimerisation chamber. Losses of material on the spinning machine, which leave it as perls, and losses due to cutting the plates at the edge, greatly influence the calibration 
uncertainty. Therefore, laboratory calibration of the measurement method was performed, where simulation of real production process was attempted.

In the following, the laboratory calibration procedure, results of laboratory calibration with water and the results of measurements in the mineral wool production plant are presented.

\section{DESCRIPTION OF LABORATORY CALIBRATION PROCEDURE}

Laboratory calibration was performed prior to the installation of system in the mineral wool production plant. Water as a media was used instead of mineral wool melt. In the mineral wool production process, calibration is impossible. The

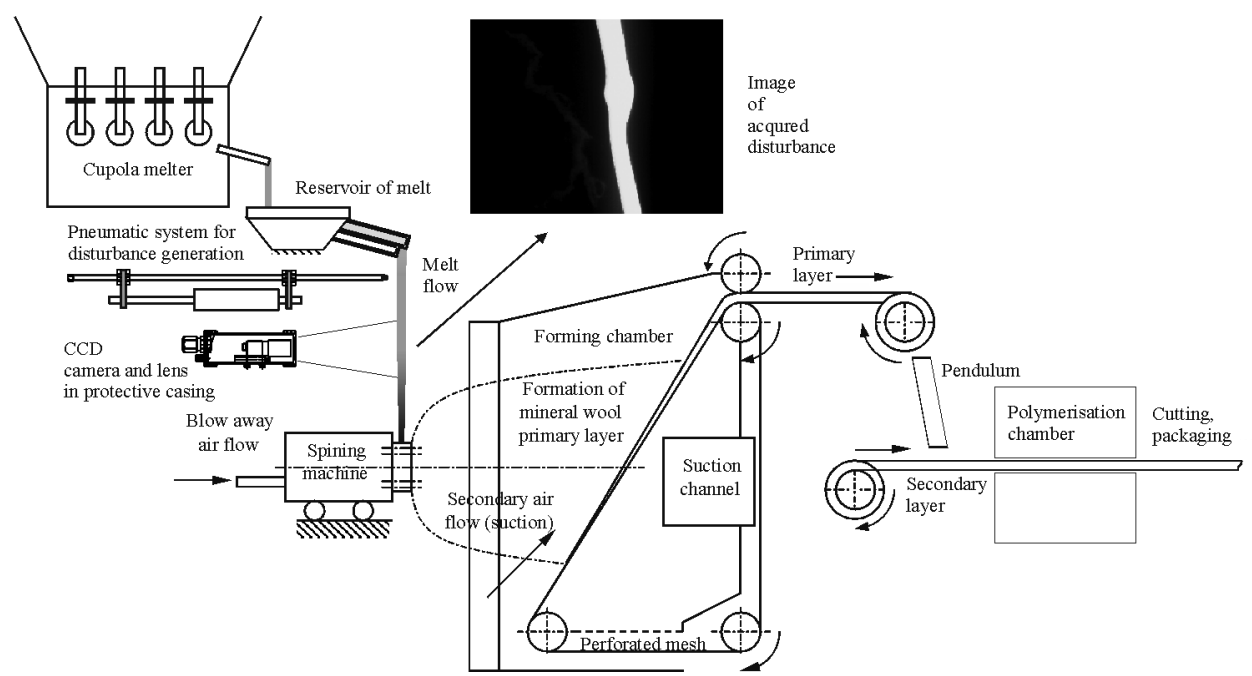

Fig. 1. Schematics of mineral wool production

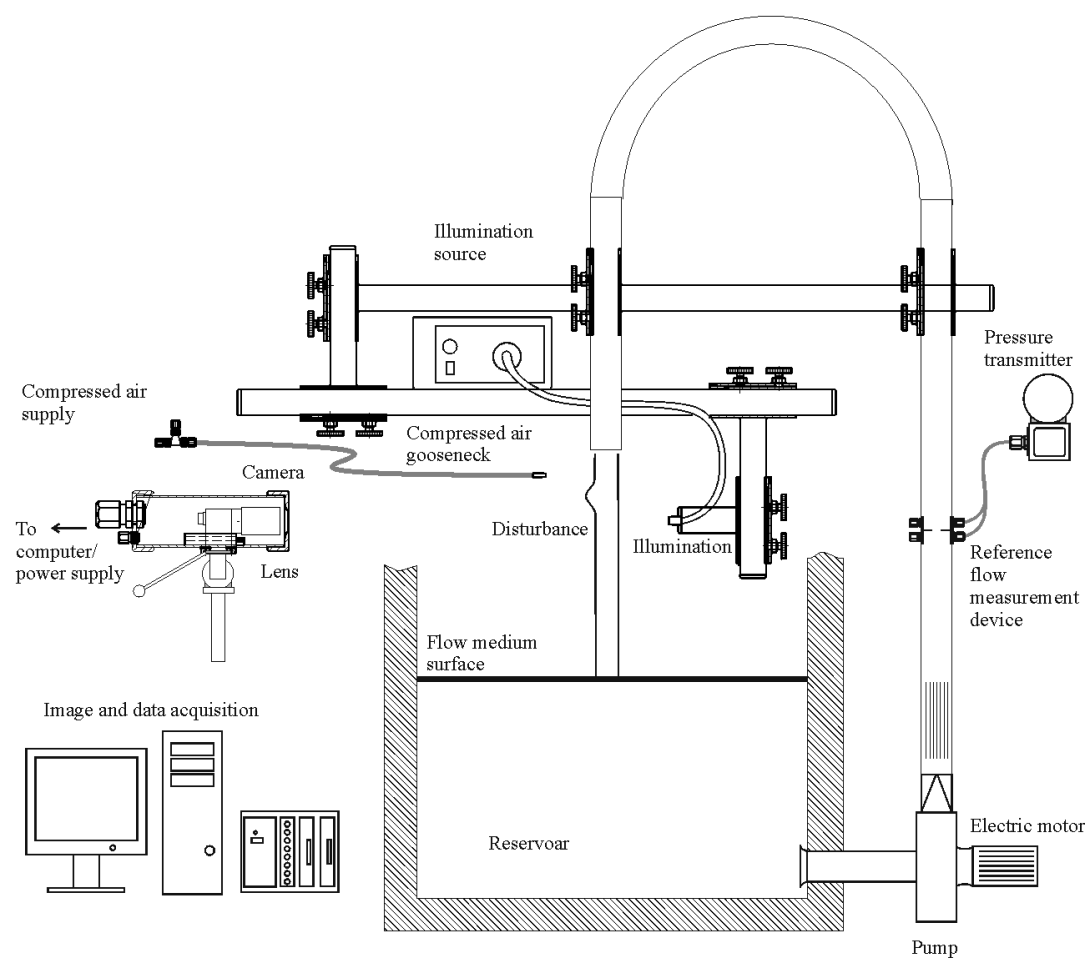

Fig. 2. Laboratory calibration layout 
laboratory experimental work was performed on the measurement system as shown in Fig. 2. It consisted of:

- pump,

- orifice and pressure transmitter for reference volume flow $Q_{\text {cal }}$ measurement,

- pneumatic system for disturbance generation,

- camera and illumination,

- signal conditioning system, data acquisition, processing and storage.

The flow medium was pumped from the reservoir by a centrifugal pump through a reference flow measurement device. The reference flow measurement $Q_{\text {ref }}$ was used for the purpose of calibration. The flow medium exited the pipe in a vertical position, which was similar to the real process where the melt flew out of the melter and directing channel. Camera observation, illumination and disturbance generation area was located $10 D_{p}$ downstream from the exit of the flow medium from the pipe. $D_{p}$ was pipe inner diameter. The flow then passed into a reservoir and was pumped again in the process of experiment.

During the experiment it was ensured that no bubbles were present in the flow. The experiment with water as a flow medium was performed. Water was colored by a yellow dye, which made it opaque and reduced reflections when illuminated, enabling adequate border detection in the image analysis process.

\subsection{Pump}

The purpose of the pump was to generate the flow simulating the outflow from the cupola melter. The pump type was centrifugal with peak volume flow $3100 \mathrm{l} / \mathrm{h}$ and peak electric power $900 \mathrm{~W}$. The pump rotational speed was varied by a voltage regulator. The shape of the pipe at the outflow was circular with inner diameter $D_{p}=17$ $\mathrm{mm}$. The flow in the camera observation region was therefore, also circular.

\subsection{Orifice and Pressure Transmitter for Reference Volume Flow Qcal Measurement}

The reference volume flow measurement was based on measuring the stagnation pressure with an orifice. It was located $20 D_{p}$ after the exit from the pump. Pipe and orifice plate inner diameter were 27.3 and $20 \mathrm{~mm}$, respectively.

Reference volume flow $Q_{c a l}$ was measured by the pressure difference on the orifice:

$$
Q_{d}=\frac{\pi d_{f}^{2} \cdot v_{d}}{4},
$$

here, $\Delta p$ is the pressure difference on the orifice, $\rho$ is density of flow medium, and $k$ is the constant of the orifice, calculated according to standard [17]. $\Delta p$ was measured with a pressure transmitter DELTBAR S PMD75 by Endress Hauser. The measurement range of the pressure transmitter was 0 to 100 mbar. Flow measurement uncertainty was largely due to flow instabilities and was estimated to $3 \%$ of the measured value.

\subsection{Pneumatic System for Disturbance Generation}

In the real process during the mineral wool production, the existence of disturbances in the melt flow is subject to operating conditions of the cupola melter. In the laboratory experiment, the disturbance was generated by a pneumatic system. Here, flow control valve and gooseneck pipe with nozzle to produce air disturbance were used. The nozzle was circular and its diameter was $3 \mathrm{~mm}$. In order to generate a disturbance of proper intensity to the flow, the direction of the nozzle and distance from the flow was adjusted by hand depending on the volume flow of the flow medium.

\subsection{Camera and Illumination}

Volume flow in the measurement region was observed by a black and white CCD camera, and an image acquisition board. SONY XCHR50 camera with acquisition frequency $f_{S}=$ $60 \mathrm{~Hz}$ and image depth 10 bits was used. The resolution of the camera was $640 \times 480$ pixels. Camera exposure time was $1 / 10000$ s. Camera pixels were square. The size of each pixel in both directions corresponded to $0.14375 \mathrm{~mm}$. Images were digitized using the National Instruments NI1410 frame grabber. Labview and Vision software from National Instruments was used for data acquisition.

The illumination in the camera measurement region was provided by a Vega 
VELUM DC 150 light source and VEGA randomized light guide. The background of the observed region was colored black, thus the calibration experiment conditions for border detection for measurement of diameter and velocity were similar to the field process conditions.

\subsection{Signal Conditioning System, Data Acquisition, Processing and Storage}

Data acquisition of the measured volume flow pressure was synchronized with image acquisition. The pressure signal was led through the SCXI 1120 conditioning device and digitized with a NI 6013 16-bit A/D data-acquisition board from National Instruments. For pneumatic valve triggering for disturbance generation, the NI 6118 digital output board was used. Labview software from National Instruments was used for data acquisition and digital triggering.

The software sequence was executed every 30 s. First with an undisturbed flow, the diameter was measured, then a disturbance was generated and a sequence of 10 consecutive images were recorded for velocity calculation, followed by an acquisition of reference data from the flow measurement device. The algorithm for velocity calculation is described in greater detail below.

\section{DESCRIPTION OF VOLUME FLOW ESTIMATION ALGORITHM}

The procedure of volume flow rate estimation consists of two parts: (1) measurements in the moment before disturbance generation, and (2) continuous measurements.

Before the moment of disturbance, generation flow diameter $d_{d f}$ is measured, while the flow is not disturbed. Velocity $v_{d}$ of the melt jet is measured at the moment of disturbance generation. Flow diameter $d_{d f}$ and velocity $v_{d}$ of the melt jet are therefore, available about the time when disturbance is triggered. The triggering of disturbance should not be performed too often as this may change the impingement point of the melt.

For continuous measurements flow diameter $d_{d f}$ is measured continuously. Mineral wool producers require information about volume flow continuously. For continuous velocity estimation the principle of contraction is used. When the melt flow velocity increases in regard to the time when the disturbance was triggered, less contraction in the flow jet is present. On the other hand, when the melt flow velocity decreases, the more contraction in the flow jet is present.

Volume flow $Q_{d}$ is estimated using image acquisition and analysis from flow diameter $d_{f}$ and velocity $v_{d}$ of the melt jet. With index $d$ measurement is denoted which is performed when the disturbance is triggered. The volume flow is measured under the assumption that the melt flow is circular (Eq. (1)):

$$
Q_{d}=\frac{\pi d_{f}^{2} \cdot v_{d}}{4} .
$$

Velocity $v_{d}$ is measured when the disturbance is triggered, while diameter measurements are available continuously. The change of velocity in intervals between disturbance generations is estimated using the contraction method. When the melt flow velocity increases, less contraction in the flow jet is present. On the other hand, when the melt flow velocity decreases, the more contraction in the flow jet is present.

The contraction volume flow $Q_{\text {contr }}$ is set to the same value as the volume flow $Q_{d}$ when the system measures the velocity of disturbance. The contraction volume flow, therefore, follows the reference volume flow when disturbance is created, while between disturbances the contraction volume flow changes continuously as the diameter and contraction of the melt flow change.

The contraction method for volume flow $Q_{\text {contr }}$ estimation was performed by the Eq. (2):

$$
Q_{\text {contr }}=\frac{K \cdot\left(e^{C}-e^{C_{d}}\right)+v_{d}}{v_{d}} \cdot \frac{d_{f}^{2}}{d_{f d}^{2}} \cdot Q_{d} .
$$

In Eq. (2), $K$ is calibration constant, $d_{f d}$ average melt diameter before the disturbance is triggered, $d$ current melt diameter, and $C$ and $C_{d}$ are current and contraction coefficient before the disturbance is triggered. Changes in viscosity or surface tension were not considered because they change very slowly in the real process. The form of equation was selected so that stable behavior was achieved, while the selection of exponential function for estimation of velocity was based 
on [18]. Ziabicki [18] presented a simple model of a steady isothermal liquid jet, where velocity of the jet is exponentially dependent on the distance from the outflow from the pipe, while the diameter of the jet decreases exponentially. Fitting the jet contour in the selected window of limited size according to [18] proved adequate in this case. Contraction coefficients $C$ and $C_{d}$ were thus calculated as damping coefficients of exponential functions fitted to the edges of current and volume flow before the triggering of disturbance. Contraction coefficients represent a measure of contraction of flow at generation of disturbance $\left(C_{d}\right)$ and at the moment of estimation of $(C)$ of volume flow. In the case of increase of the volume flow rate compared to the time when disturbance was triggered $\left(C>C_{d}\right)$ the value in parentheses is positive, while in the case of reduction of volume flow rate $\left(C<C_{d}\right)$ it is negative.

Constant $K$ of value 0.1 was used. The extensive work has not been performed to justify the selection of the constant. It is assumed that the estimation of $Q_{\text {contr }}$ using the above procedure is appropriate when the velocity of flow changes slowly as this is the case during the mineral wool production process. Laboratory calibration with water volume flow was constant during particular measurement.

\subsection{Measurements of Melt Flow Diameter $d_{f}$}

Melt flow diameter $d_{f}$ was measured in an image window of approximate size $6 d \times 3 d$. In the window 300 horizontal intensity profiles were calculated. In each intensity profile, thresholding was performed, and the diameter was estimated based on the pixels above the threshold. Threshold value 500 was used for the 10-bit image. Diameter detection algorithm included sub-pixel accuracy. To calculate the diameter $d_{f}$ of melt flow in the window, all 300 diameters in a single image were averaged. In addition, diameters in the last 10 consecutive images were averaged.

Here, measurement with only one camera was used for calibration and measurements in the plant. For calibration use of one camera is adequate as outflow pipe as seen in Fig. 2 has circular cross section. In the plant, situation is different, the melt flows from a channel, which is cooled with water. Due to solidified melt on the cool edges, the melt flow regularly changes its cross section at the position of the channel. Melt flow does change cross section to circular for some length. Due to a non-circular cross section, additional measurement uncertainty appears. Use of two or more cameras could improve this. If we estimate, that melt flow cross section has an elliptical cross section, they should be mounted in both principal axes of the ellipse for the case of two cameras.

Further sources of melt flow diameter df measurements uncertainty are limited resolution and field depth of the camera. Camera resolution was selected with compromise to the frame rate to enable $60 \mathrm{~Hz}$ frame rate required for flow velocity measurement. For an estimation of diameter, sub pixel accuracy was used and measurement uncertainty was therefore, much reduced. Field depth during calibration did not contribute to measurement uncertainty because the flow did not move and was properly illuminated, while during experiments in the mineral wool production plant the cameras lens was fully closed due to very high light intensity of the melt flow. Therefore, the field of depth was much higher than the maximum movement of the melt flow.

\subsection{Measurements of Melt Flow Velocity $v_{d}$}

Melt flow velocity $v_{d}$ was measured at the moment of triggering of melt volume flow disturbance. Velocity $v_{d}$ was estimated by observing propagation $l$ of disturbance in two consecutive images recorded in time interval $\Delta t$, that is $v_{d}=l / \Delta t$. Time interval $\Delta t$ between two consecutive images was $1 / 60 \mathrm{~s}$. A sequence of 10 image pairs was recorded after the disturbance was generated. The timing of disturbance generation and image acquisition was synchronized so that the acquired image pairs included the entire flow disturbance propagation.

In each consecutive image pair, intensity profile was measured in each line of each image, and corresponding image diameters were calculated using the same thresholding method as for melt flow diameter measurement. Thus, diameters $x(i)$ for the first and $y(i)$ for the second image in the image pair were calculated. $i$ is the index of lines in diameter series. 
Cross correlation method was used to calculate the propagation $l$ of disturbance of every image pair. The cross correlation $r(k)$ was calculated for every shift $k$ between both diameter series as shown by Eq. (3):

$$
r(k)=\frac{\sum_{i}(x(i) \cdot y(i-k))}{\sqrt{\sum_{i}(x(i))^{2}} \sqrt{\sum_{i}(y(i))^{2}}} .
$$

For a certain value of shift $k$, where cross correlation $r(k)$ is maximal, shift $k$ between two consecutive images represents propagation $l$ of disturbance. For propagation $l$ of disturbance, averaging over all 10 acquired image pairs was used. By doing so, only one velocity filed vector per image pair was calculated. This is different from most of other PIV applications and a serious limitation of the measurement procedure. However, this is necessary due to a low amount of details with melt flow fully saturated, present on individual image pairs. One possibility to improve the method would be to split the melt flow in a series of vertical sections and calculate velocity, which might require the use of filters on the camera lens. This would also mean that better representation of velocity field inside the melt flow could be achieved, as section of the melt near the channel outflow may slower than in other sections.

\section{DESCRIPTION OF MINERAL WOOL PRODUCTION PLANT MEASUREMENTS}

Experimental work in a mineral wool production plant was based on comparing melt volume flow with the amount of produced mineral wool insulation material. In the field experiments, the manufactured insulation material on the production line was weighed after the collection chamber. The weakness of this method is that a part of melt is not transformed into fibres and leaves the spinning machine as perls. These perls fall down due to a high specific weight before they reach the collecting chamber and do not contribute to the weight of the insulation material. In addition, the panels are cut from side to the desired size and some of the insulation material is therefore not included in calibration. During the experiment no production parameters were changed, so it was estimated that the amount of losses is constant. The measured weight of melt flow in the selected time interval before the spinning machine is, therefore, always higher than the weight of the insulation material after the collecting chamber. Volume flow of melt was measured using the procedure described in section 2. To calculate the mass flow $Q_{m}=r \cdot Q_{v}$ for density $\rho$ of melt, the value provided by the manufacturer of mineral wool was used, calculated according to the algorithm by [1], which takes into consideration the temperature and chemical composition of raw materials.

In order to measure the weight of panels, a mechanical weighing device was used. Every 60 $\mathrm{s}$, four panels of total size $2 \times 1 \mathrm{~m}$ were removed from the production line and weighed.

\section{RESULTS AND DISCUSSION}

In this section, the results of laboratory calibration and measurement results in a mineral wool production plant are presented.

\subsection{Laboratory Calibration}

The description of laboratory calibration is presented in section 2. For the selection of volume flow, the rotational speed of the pump was regulated by changing the voltage of the electric motor. Fig. 3 show calibration diagrams for water. In each measurement point, 50 measurements were evaluated. $\bar{Q}_{\text {contr }}$ and $\bar{Q}_{c a l}$ are average values of $Q_{\text {contr }}$ and $Q_{\text {cal }}$ measurements in each measurement point. The calibration diagram for water gives regression coefficients $R^{2}=0.99$.

Throughout the paper the following definition of regression coefficient is used:

$$
R=\frac{n \sum_{1}^{n} x_{i} y_{i}-\sum_{1}^{n} x_{i} \sum_{1}^{n} y_{i}}{\sqrt{n \sum_{1}^{n} x_{i}^{2}-\left(\sum_{1}^{n} x_{i}\right)^{2}} \sqrt{n \sum_{1}^{n} y_{i}^{2}-\left(\sum_{1}^{n} y_{i}\right)^{2}}} .
$$

However, the weakness of the measurement method is that single measurements of $Q_{\text {contr }}$ have relatively high deviation around the average.

Correlations may change with experimental conditions; among them are intensity of disturbance generation, illumination, 
diameter of the pipe and velocity of the flow in the observation window etc. In addition correlation may change if larger or smaller interval of volume flows is selected. Influence of experimental conditions change was not investigated, and the measurements were performed at pre-set values. Among influent parameters, only disturbance generation was adjusted. At single measurement point at selected volume flow, all experimental conditions were constant.

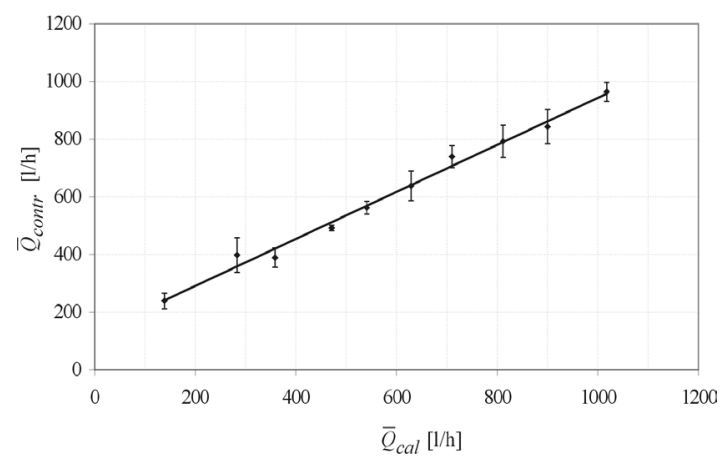

Fig. 3. Calibration diagram for water, error bars show standard deviation of measurements of $Q_{\text {contr }}$

In the production process, melt flow velocities and volume flows depend on the pressure inside the melter, the height of the measurement position downstream, the melt flow and on the presence of hardened melt on the outflow from the directing channel. The disagreement between the measured volume flow $Q_{\text {contr }}$ and the calibration volume flow $Q_{\text {cal }}$ arises from the unknown location of velocity measurement, interaction with the blowing compressed air for disturbance generation and a limited number of image pairs for correlation analysis.

The exact location of the measurement of velocity according to Eq. (1) is not known and can be anywhere in the image observation window. The correlation analysis technique (Eq. (3)) can only provide instantaneous velocity inside the correlation observation area. When disturbance is detected in the lower part of the observation window, the measured velocity is higher, while in the case when the disturbance is detected in the upper part of the observation window, the velocity is lower. For the diameter of melt flow, the measured average middle diameter was used.
One possible solution of this problem is to use a narrow correlation calculation window, however, it would be difficult to trigger the disturbance in two consecutive images. In addition, more image pairs and more disturbances would be required.

The second source of measuring uncertainty of reference volume flow is the change in the velocity of the parts of melt flow due to the interaction with the blowing compressed air. In this case, the direction of blowing with compressed air was perpendicular to melt flow in order to minimize this influence. In addition, as the smallest disturbance possible was used, which was still adequate for good velocity detection using correlation algorithm.

The third source of measurement uncertainty is the limited number of acquired image pairs for each disturbance generation. For this analysis, 10 image pairs were acquired. The selection of the number of acquired image pairs was, however, influenced by the limited time available for disturbance generation.

To a smaller degree, the instability of pump operation also increases measurement uncertainty of volume flows $Q_{d}$ and $Q_{\text {contr }}$.

Fluids with higher viscosity are less susceptible to pulsations with compressed air, and the spraying of parts of fluid is less probable. A distinctive feature of Fig. 3 is that fitted calibration lines do not cross the starting point of the diagram. Flow instabilities on the edge of the pipe add a certain amount to the diameter of the flow regardless of position.

Fig. 4 shows flow diameters $d_{f}$ for experiments with water. Diameters $d_{f}$ were calculated from images as mean diameters in the observation window. The measurements performed with water have regression coefficient $R^{2}=0.96$. Results show that in the observation region the diameter $d_{f}$ of the flow is linearly dependant on the volume flow. On the other hand, the diameter of the flow in laboratory calibration was limited by the diameter of outflow pipe. It therefore be supposes that linear relationship can only be expected at low volume flows. Some saturation can also be seen at volume flows above 1000 l/h in Fig. 4. The water jet at the exit from the pipe has fixed diameter, and in the position some diameters from the outlet downwards the flow is accelerated and thinned. Thus, the flow can not 
have zero diameter and there is no requirement for plots to pass through origin of Fig. 3 maintaining linear behavior observed at higher volume flows. Diameter measurement is reasonable down to the limit diameter where the flow is not sustained anymore and droplets are formed because of surface tension.

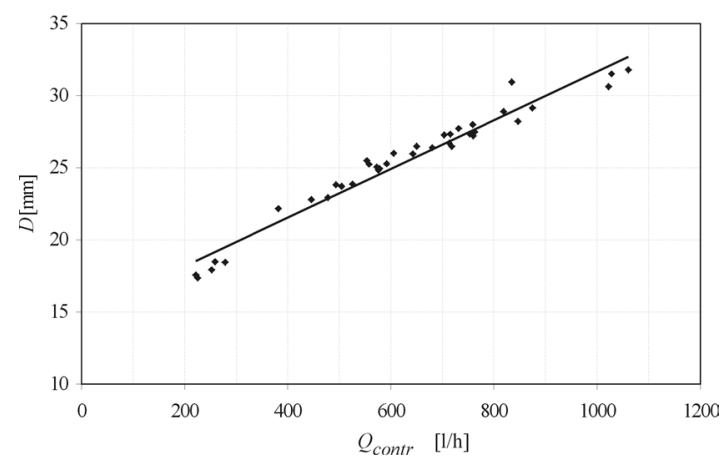

Fig. 4. Flow diameter diagram for water

\subsection{Production Process Measurements}

Production process measurements were performed during the mineral wool production process. Fig. 5 shows image pairs acquired during field experiments. Two cases of melt flows are shown: with disturbance generated using pneumatic system (Fig. 5a) and without disturbance (Fig. 5b). Both cases show two images recorded at a time interval $\Delta t=1 / 60 \mathrm{~s}$. The first acquired image is shown above. Images were taken in a real production process.

Fig. 6 shows the results of the field measurement of the weight of melt flow in the selected time interval and produced insulation material after the collecting chamber. The agreement between the two variables is very good.

The measurements using the melt flow measurement technique successfully follow low frequency variations of the weight of insulation panels. This is suitable for the regulation of the production line speed to ensure the optimal density of the product and for the regulation of melter. The measurement results regarding the weight of panels show less high frequency variations than measurements of melt flow.

During the measurement it was ensured by continuous and careful cleaning of the flow channel that the flow channel from the melter was free of solidification remains. The melt solidifies on water-cooled walls of the channel. Thus, it was possible to maintain the cross-section of the melt flow in the measurement position as circular as possible. Measurements of melt flow without cleaning the flow channel had much higher measurement uncertainty (results not shown). Results show that use of one camera is adequate, if the flow channel is carefully cleaned.

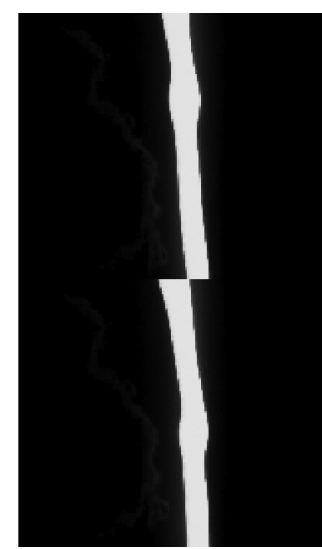

a)

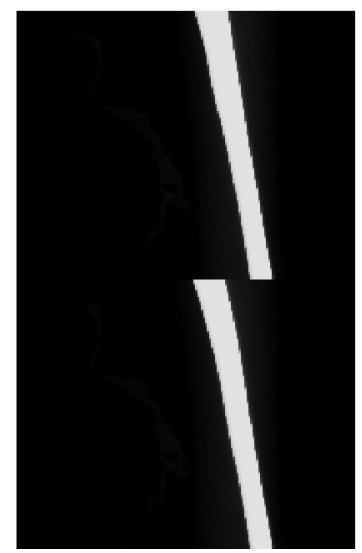

b)
Fig. 5. Propagation of disturbance;

a) disturbance generated by pneumatic system; b) no disturbance is present

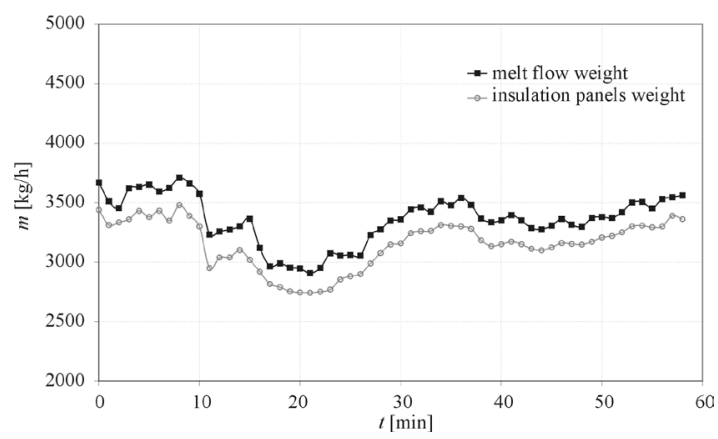

Fig. 6. Results of field measurements, comparison of measurement results regarding the weight of melt flow in the selected time interval and weight of produced insulation panels after the collecting chamber

It is assumed that the results of melt flow measurement agree well with the results of panel weighing also because of the high viscosity of melt. High viscosity of melt enabled 
the generation of adequate disturbances and prevented the spraying of melt. Thus, the results of field measurements are better than the results of calibration in the laboratory, however, the validation procedure for both cases was different.

\section{CONCLUSIONS}

A method for measuring melt volume flow for mineral wool production has been presented. The method for measuring melt volume flow by using a vision system and disturbance generation proved successful in a field experiment. During laboratory calibration the variations of individual measurements had high standard deviations, while the average values were very accurate. However, several problems should still be addressed; size of velocity estimation region, a change of flow velocity due to pneumatic disturbance generation, and the increase in measurement uncertainty due to solidification remains on the outflow directing channel.

\section{REFERENCES}

[1] Širok, B., Blagojević, B., Bullen, P. (2008). Mineral wool, production and properties, Woodhead Publishing in Materials, Cambridge.

[2] Improvements in or relating to the production of mats or the like of mineral wool, international patent GB822528 (1959). ROCKWOOL AB, application number: GB19570015575 19570516.

[3] Širok, B., Mihovec, B., Bradeško, F., Možina, P. (2005). Conveyor scale for controlling rock wool surface density European Patent Nr. EP 1194754 B1: Bulletin 2005/35,European Patent Office, Munich.

[4] Hočevar, M., Širok, B., Blagojević, B. (2005). Mineral wool production monitoring using neural networks. International Journal of Information Technology, vol. 11, p. 64-72.

[5] Raffel, M., Willert, C.E., Wereley, S.T., Kompenhans, J. (2007). Particle Image Velocimetry, A Practical Guide. Springer, Heidelberg.
[6] Adrian, R.J. (2005). Twenty years of particle image velocimetry. Experiments in Fluids, vol. 39, p. 159-169.

[7] Tokumaru, P.T., Dimotakis, P.E. (1995). Image correlation velocimetry. Experiments in Fluids, vol. 19, no. 1, p. 1-15.

[8] Cheng, W., Murai, Y., Sasaki, T., Yamamoto, F. (2005). Bubble velocity measurement with a recursive cross correlation PIV technique. Flow Measurement and Instrumentation, vol. 16, no. 1, p. 35-46.

[9] Zosel, J., Guth, U., Thies, A., Reents, B. (2003). Flow measurements in micro holes with electrochemical and optical methods. Electrochimica Acta, vol. 48, p. 3299-3305.

[10] Hann, D.B., Greated, C.A. (1997). The measurement of flow velocity and acoustic particle velocity using particle-image velocimetry. Measurement Science \& Technology, vol. 8, p. 1517-1522.

[11] Patte-Rouland, B., Lalizel, G., Moreau, J., Rouland, E. (2001). Flow analysis of an annular jet by particle image velocimetry and proper orthogonal decomposition. Measurement Science \& Technology, vol. 12, p. 1404-1412.

[12] Hu, H., Saga, T., Kobayashi, T., Taniguchi, N. (2002). Simultaneous measurements of all three components of velocity and vorticity vectors in a lobed jet flow by means of dual-plane stereoscopic particle image velocimetry. Physics of Fluids, vol. 14, p. 2128-2138.

[13] Sakakibara, J., Wicker, R.B., Eaton, J.K. (1996). Measurements of the particle-fluid velocity correlation and the extra dissipation in a round jet. International Journal of Multiphase Flow, vol. 22, p. 863-881.

[14] Beck, M.S., Plaskowski, A. (1987). Cross correlation flowmeters - their design and application. Adam Hilger, Bristol.

[15] Carlson, J., Ing, R.K. (2003). Ultrasonic speckle correlation imaging of 2D particle velocity profiles in multiphase flows. Flow Measurement and Instrumentation, vol. 14, no. 4-5, p. 193-200.

[16] Takamoto, M., Ishikawa, H., Shimizu, K., Monji, H., Matsui, G. (2001). New measurement method for very low liquid flow rates using ultrasound. Flow 
Measurement and Instrumentation, vol. 12, no. 4, p. 267-273.

[17] ISO 5167 (2003). Measurement of fluid flow by means of pressure differential devices inserted in circular cross-section conduits running full. International Organization for Standardization, Geneva.

[18] Ziabicki, A. (1976). Fundamentals of fibre formation. Wiley, New York. 